\title{
AUSTRALIA Y NUEVA ZELANDA
}

Greta Bucher

Universidad Iberoamericana

\section{INTRODUCCIÓN}

Australia y Nueva Zelanda, dos países vecinos ubicados en la parte sudoeste del océano Pacífico, son conocidos generalmente por sus abundantes recursos naturales, su gran calidad de vida y su ausencia de corrupción. La relación entre ambos países, también conocida como Trans-Tasman (relaciones a través del mar de Tasmania), es muy cercana, pues no sólo comparten lazos culturales e históricos debido a una herencia colonial británica común, sino que son parte de la Mancomunidad de Naciones, por la cual reconocen a la reina Isabel II de Inglaterra como jefa de Estado. Asimismo, mantienen estrechas relaciones económicas y comerciales, las cuales son reconocidas como de las más amplias y compatibles del mundo según el propio gobierno neozelandés (New Zealand Foreign Affairs and Trade, 2018). Desde 1983, el comercio bilateral ha aumentado de manera constante; actualmente Australia sigue siendo el principal socio comercial de Nueva Zelanda.

A su vez, Australia y Nueva Zelanda son aliados militares debido al Acuerdo Comercial de Relaciones Económicas más Estrechas (ANZCERTA, por sus siglas en inglés), también conocido como el Acuerdo de Relaciones Económicas más Estrechas (CER, por sus siglas en inglés), y al Acuerdo Relaciones de Defensa más Estrechas (CDR, por sus siglas en inglés), que ambas naciones mantienen desde 1983 y 1991, respectivamente (New Zealand Foreign, 2018).

Ambos países son democracias representativas, con gobiernos parlamentarios; sin embargo, durante el último lustro Australia ha experimentado cierta inestabilidad política, algo que no ha sido el caso de Nueva Zelanda. Con la salida del entonces primer ministro 
Malcolm Turnbull en agosto de 2018, debido a una lucha interna en el Partido Liberal de Australia, Scott Morrison se convirtió en el quinto primer ministro en los últimos cinco años. Asimismo, durante 2018 Australia sufrió una serie de escándalos políticos que, junto con la renuncia de Turnbull a su escaño en la Cámara de Representantes, llevaron a convocar a elecciones suplementarias en dos ocasiones.

Si bien Nueva Zelanda puede considerarse más estable en términos políticos -ha tenido tres primeros ministros de 2008 a la fecha-, no estuvo exenta de ciertas controversias y escándalos políticos durante 2018. Uno de los temas más escandalosos surgió luego del anuncio del embarazo y consecuente incapacidad por maternidad de su recién instaurada primera ministra laborista Jacinda Ardern, quien logró arrebartarle el liderazgo político al Partido Nacional de Nueva Zelanda — en el poder desde 2008 - tras lograr formar un gobierno de coalición a finales de 2017, con el apoyo de Nueva Zelanda Primero y del Partido Verde.

Otros temas relevantes fueron la huelga de maestros, la primera desde 1994, y el debate que surgió en torno a la propuesta de ley presentada en junio de 2017 por el líder del partido político ACT, David Seymour, llamada End of Life Choice (Elección de fin de vida), cuyo objetivo es legalizar la eutanasia voluntaria. El tema cobró importancia dado que es la primera vez que una propuesta de dicho tema es aprobada en primera instancia por el Parlamento. El proyecto seguirá un proceso en el cual se tendrá que revisar y debatir dos veces más antes de ser aprobada, lo cual se espera suceda a principios de 2019.

A continuación presentaré éstos y otros hechos relevantes en materia de política nacional, política exterior, económica y comercial, que tuvieron lugar durante 2008 tanto en Australia como en Nueva Zelanda.

\section{POLÍTICA NACIONAL}

La inestabilidad ha sido una característica de la política nacional australiana durante los últimos diez años. En 2018, dicha inestabilidad se incrementó con la salida forzada de Malcolm Trunbull, líder del gobierno desde 2015, debido a una lucha interna del Partido Liberal. Tras una segunda votación entre los miembros del partido, ${ }^{1}$ Scott Morrison,

\footnotetext{
${ }^{1}$ En la primera votación, Julie Bishop, ministra de Relaciones Exteriores, quedó fuera de la contienda.
} 
previamente jefe del Tesoro, logró vencer al contendiente, Peter Dutton, ministro del Interior, por 45 votos contra 40. De tal modo, Morrison logró convertirse en el 30o primer ministro de Australia, el quinto en el último lustro. ${ }^{2}$

La inestabilidad al interior del Partido Liberal podría resultar contraproducente para el propio gobierno de coalición liberal-nacional, el cual gobierna con una mayoría de tan sólo un escaño. La renuncia de Turnbull a su cargo en el Parlamento al poco tiempo de ser depuesto, y la consecuente convocación a elecciones suplementarias en el distrito de Wentworth, Nueva Gales del Sur, en octubre para elegir a su sustituto, ${ }^{3}$ podrían ocasionar que el gobierno pierda dicha mayoría y obligarlo a convocar a elecciones federales anticipadas, motivo por el cual existe el riesgo de que la coalición pierda su mandato.

Otras cuestiones también contribuyeron a la inestabilidad política de Australia durante 2018. A inicios de mayo, tres diputados del Partido Laborista Australiano — principal partido de oposición - y una diputada del partido Centre Alliance se vieron obligados a renunciar por tener doble nacionalidad: Justine Keay, Josh Wilson, Susan Lamb y Rebekha Sharkie, respectivamente (Baidawi, 2018); ${ }^{4}$ esto provocó la necesidad de convocar a elecciones suplementarias el 28 de julio, lo cual también ponía en riesgo el futuro del gobierno australiano. Si bien estas renuncias demuestran cierta inestabilidad en la política australiana, resulta relevante resaltar que los resultados de las elecciones suplementarias no modificaron la composición de la Cámara de Representantes: tanto el Partido Laborista de Australia como el partido Centre Alliance conservaron sus escaños (Australia Electoral Commission, 2018).

Ahora bien, a pesar de que los criterios de elegibilidad para ser miembro del Parlamento Federal establecidos en la Sección 44 de la Constitución Australiana son claros, ${ }^{5}$ situaciones semejantes a las anteriores han ocurrido quince veces en menos de un año (Baidawi, 2018). Otro caso semejante, fue por ejemplo, el de la senadora laborista Katy Galler, quien una vez que el Tribunal Supremo dictaminó que no era elegible para permanecer en el Parlamento ya que no había renunciado a su ciudadanía británica antes de la elección federal de 2016,

\footnotetext{
${ }^{2}$ Julia Gillard (junio de 2010-junio de 2013), Kevin Rudd (junio de 2013-septiembre de 2013), Tony Abbott (septiembre de 2013-septiembre de 2015) y Malcolm Turnbull (septiembre de 2015-agosto de 2018).

${ }^{3}$ De acuerdo con la Comisión Electoral Australiana, una elección parcial debe realizarse cada vez que exista una vacante en la Cámara de Representantes.

${ }^{4}$ Un quinto diputado laborista, Tim Hammond, decidió dimitir por motivos familiares, de ahí que el 28 de julio se realizaron elecciones parciales en cinco divisiones electorales.

${ }^{5}$ La Sección 44 establece cinco criterios según los cuales una persona no puede ser elegible a ocupar un puesto en el Parlamento Federal; contar con doble nacionalidad es uno de ellos.
} 
provocó las renuncias voluntarias de los cuatro diputados arriba mencionados (Baidawi, 2018).

Dada la relevancia del tema, durante 2018 se debatió la posibilidad de modificar la Sección 44 de la constitución australiana, e incluso de celebrar un referéndum para ello. Sin embargo, debido a la proximidad de las elecciones suplementarias del 28 julio de 2018 y de las elecciones federales de $2019,{ }^{6}$ el gobierno de Turnbull consideró más oportuno introducir medidas temporales y formatos adicionales con el objetivo de mejorar el proceso de nominación y de certificar la nacionalidad y otras descalificaciones de los cantidados, así como no convocar a un plebicito en la materia (Doran, 2018).

Otra renuncia importante en 2018 fue la del entonces primer viceministro y líder del Partido Nacional de Australia, Barnaby Joyce, debido a un escándalo sexual. En febrero de 2018 Joyce fue sustituido por Michael McCormack en ambos puestos; sin embargo, no renunció a su posición en la Cámara de Representantes, ya que de hacerlo le hubiera restado a Turnbull el asiento que le daba al gobierno de coalición la mayoría necesaria para gobernar, riesgo que se tomó luego del golpe interno contra Turnbull.

Nueva Zelanda, por su parte, vivió una transición política en octubre de 2017, cuando la laborista Jacinda Ardern se convirtió en la 40a primera ministra de Nueva Zelanda al formar un gobierno de coalición con el apoyo del partido Nueva Zelanda Primero y del Partido Verde, dos pequeños partidos políticos que se volvieron relevantes en la última elección dado que ni el partido Laboral ni el Nacional — los partidos políticos más relevantes del país - consiguieron la mayoría necesaria para formar gobierno. Ardern es la tercera mujer en ocupar el cargo y, con 37 años de edad, es la líder neozelandesa más joven en los últimos 150 años. Su victoria significó el fin del mandatado de casi diez años del Partido Nacional de Nueva Zelanda.

Por otra parte, algunas de las prioridades del gobierno de Ardern son restaurar el financiamiento del sistema de salud para permitir el acceso universal, asegurar que la población cuente con buenas condiciones de vida, tomar medidas contra la pobreza infantil, limpiar los ríos, redoblar los esfuerzos para enfrentar el cambio climático y realizar la transición a una economía baja en carbono (Ardern, 2017). Asimismo, concentrar sus

${ }^{6}$ La única excepción sería que el primer ministro Turnbull volviera a implementar una doble disolución del Parlamento. 
esfuerzos en el desarrollo económico sostenible, apoyando las economías regionales, aumentando las exportaciones y los salarios, y reduciendo la desigualdad (Jacinda Ardern, 2017).

Como primera ministra electa, Ardern, y el líder de Nueva Zelanda Primero Winston Peters — quien se convirtió en primer viceministro y ministro de Asuntos Exteriores-, firmaron un compromiso en Wellington. Según el mismo, la prosperidad económica deberá ir acompañada de una responsabilidad ambiental, y se deberá: prohibir a compradores extranjeros adquirir viviendas en Nueva Zelanda, reducir la inmigración en hasta 30000 personas anualmente, y revisar y reformar la Ley del Banco de la Reserva (Ainge, 2017).

Ardern firmó también un compromiso con el Partido Verde, que incluye acuerdos como: transformar la economía para conseguir un nivel neto de cero emisiones para 2050; realizar una inversión sustancial en opciones alternativas de transporte, como senderos y carriles para bicicletas; reformar el sistema de bienestar, y proteger a las 3000 especies neozelandesas que se encuentran en peligro de extinción (Ainge, 2017).

Junto con la controversia que causó el anuncio de su embarazo en enero de 2018 y posteriormente su incapacidad una vez que nació su primer hijo, uno de los principales retos que enfrentó Ardern durante el 2018 fue la huelga de profesores de primaria del 15 de agosto, la primera desde 1994, liderada por el sindicato del Instituto Educativo de Nueva Zelanda. Algunas de las demandas del profesorado fueron: aumento salarial de por lo menos $16 \%$ en un plazo de dos años; aulas con un número menor de estudiantes; escuelas correctamente equipadas, con los recursos necesarios para impartir clases, y coordinadores de educación especial en todos los centros de estudio.

La contraoferta del Ministerio de Educación llegó a mediados de septiembre: aumento salarial de 3\% anual durante los próximos tres años; la contraoferta no incluyó cambios en el número de estudiantes ni apoyo adicional para niños con necesidades especiales. Así que luego de una votación secreta de los miembros del sindicato, dicha oferta fue rechazada el 25 de septiembre. El curso de acción será decidido el 30 de septiembre durante la conferencia anual del Instituto Educativo.

Otro tema que generó debate en la política nacional neozelandesa fue el provocado por el proyecto de ley presentado en junio de 2017 por el líder del partido político ACT, David 
Seymour, llamado End of Life Choice (Elección de fin de vida), cuyo objetivo es legalizar la eutanasia voluntaria, aunque en el documento no se menciona dicha palabra de manera específica. De acuerdo con la propuesta, sólo un enfermo terminal con una esperanza de vida de máximo seis meses, o una persona que padezca una condición médica grave e irremediable, se encuentre en un estado avanzado de deterioro irreversible, experimente un sufrimiento insoportable que no pueda aliviar, sea mayor de edad y no padezca de sus facultades mentales, podría solicitar asistencia médica para morir.

Si bien no es la primera vez que se debate legalizar la eutanasia en Nueva Zelanda, este proyecto cobró importancia en 2018 debido al debate que generó. A diferencia de los intentos previos en 1995 y 2003, que no contaron con el apoyo inicial del Parlamento, en diciembre de 2017, luego de revisar y de debatir la propuesta, ésta se aprobó en primera instancia, con 76 votos a favor y 44 en contra (Murphy, 2017). Ahora, el Comité Selecto de Justicia, presidido por el diputado laborista Raymond Huo, celebrará audiencias y recibirá presentaciones públicas. Posteriormente el proyecto seguirá un proceso en el cual se tendrá que revisar y debatir dos veces más. Se espera que dicha propuesta se apruebe a inicios de 2019, ya que, según las encuestas, cuenta con el 75\% de aprobación de la población (Murphy, 2017).

\section{POLÍTICA EXTERIOR}

A pesar de los lazos históricos que ambos países mantienen con el Reino Unido, la región Asia-Pacífico se ha vuelto de vital relevancia en cuestiones políticas, económicas, comerciales y de seguridad nacional, tanto para Australia como para Nueva Zelanda.

A finales 2017, el gobierno australiano publicó los objetivos de su política exterior para los siguientes años, entre los que destacan: promover una región Indo-Pacífico abierta, inclusiva y próspera; ofrecer más oportunidades para la iniciativa privada a escala mundial, opuesta al proteccionismo; garantizar la seguridad de los australianos frente al terrorismo; promover y proteger las normas internacionales que respaldan la estabilidad y la prosperidad (Australian Government, 2017).

Debido a lo anterior, y dadas las implicaciones que podría tener sobre la posición internacional de Australia, el lanzamiento de la Estrategia de Exportaciones de Defensa del 
gobierno de Turnbull causó cierta polémica al interior del país. En términos generales, la estrategia plantea un objetivo para 2028: aumentar las exportaciones en materia de defensa. Se pretende que Australia se convierta en uno de los diez principales exportadores de armas del mundo, con el objetivo de crear una industria militar más sostenible y competitiva capaz de soportar las necesidades de la Fuerza de Defensa Australiana (Department of Defence. Australian Government, 2018). Asimismo, la estrategia establece un sistema integral para planear, guiar y medir los resultados de las exportaciones en materia de defensa, mediante la creación de una Oficina de Exportación de Defensa Australiana.

Ahora bien, de acuerdo con la información publicada por el Departamento de Defensa australiano, las exportaciones en esta materia serán una valiosa contribución para la comunidad internacional, y equilibrarán las relaciones bilaterales y las obligaciones internacionales de Australia. Al mismo tiempo, el gobierno señaló que la estrategia impulsará la industria nacional, la creación de empleos y el crecimiento económico del país. Para cumplir con lo propuesto, se destinarán alrededor de 14.6 millones de dólares adicionales entre 2018 y 2019 (Department of Defence, 2018).

Aun cuando la estrategia establece que Australia seguirá comprometida con sus obligaciones internacionales sobre el control de armas —el cual regula la exportación de bienes, software y tecnología mediante controles de exportación determinados por el Departamento de Defensa-, ciertos actores nacionales como el Australian Council for International Development consideran que la estrategia es una prioridad incorrecta. Ellos afirman que Australia debe apoyar la paz y el desarrollo mundial y no contruibuir al conflicto mediante la exportación de armamento (Australian Council for International Development, 2018).

En contraposición, Australia presentó en julio su primera Revisión Nacional Voluntaria en cuanto a los Objetivos de Desarrollo Sostenible, ante el Foro Político de Alto Nivel sobre Desarrollo Sostenible de las Naciones Unidas. Éste es un informe relacionado con el esfuerzo global australiano por cumplir con la Agenda 2030. El informe tiene un enfoque narrativo y aborda cada uno de los objetivos de desarrollo sostenible. Un anexo enumera las políticas nacionales existentes para el logro de dichos objetivos. Además, el informe aclara que están en marcha muchas medidas en los ámbitos estatal, territorial y municipal, e incluye los 
esfuerzos del sector empresarial, la sociedad civil, el mundo académico, las comunidades y los individuos (Australian Government, 2018).

Los temas más relevantes del informe se relacionan con los objetivos del país relacionados con salvaguardar y cuidar el suministro del agua y el medio ambiente. Asimismo, se señala que el gobierno ha implementado políticas para mejorar las condiciones de vida en las ciudades, lo que incluye inversiones en infraestructura y reformas mediante el proyecto City Deals, al tiempo que está trabajando para financiar y poner en marcha un Esquema Nacional de Seguro de Incapacidad.

De acuerdo con el informe, el gobierno estableció el compromiso de reconocer a los pueblos aborígenes y a los isleños del Estrecho de Torres de manera constitucional. Aun cuando no existe un objetivo específico para ello, los 17 Objetivos de Desarrollo Sostenible resultan relevantes para las necesidades y aspiraciones de estos pueblos, ya que el concepto guía de "cuidar el país" no sólo incorpora la gestión ambiental, sino también el bienestar sociopolítico, cultural, económico, físico y emocional de la población. Sin embargo, de acuerdo con información de The Economist, el gobierno de coalición ofreció celebrar un referéndum sobre dicho "reconocimiento constitucional" (Australia's States Offer, 2018), por lo que el tema sigue pendiente.

Nueva Zelanda, país que se define asimismo como multilateral, imparcial e independiente, actualizó en 2018 un informe que se publicó en febrero de 2017, el Strategic Intentions 2017-2021, en el cual establece los objetivos estratégicos y las prioridades del gobierno en materia de política exterior para el periodo señalado.

El nuevo documento, Strategic Intentions 2018-2022, tiene como objetivo establecer un horizonte de planificación de diez años, en el cual se plasman los objetivos y resultados en materia de política exterior que el gobierno considera estratégicos y que espera alcanzar durante la siguiente década. Éstos son: fortalecer, proteger y utilizar las reglas e instituciones internacionales para perseguir los valores e intereses de Nueva Zelanda; aumentar los rendimientos económicos sostenibles y fortalecer el comercio, la inversión y otras conexiones internacionales; posicionar a Nueva Zelanda como un socio activo e integral en la región Asia-Pacífico, a fin de promover la estabilidad regional y la integración económica; promover soluciones internacionales sostenibles para los retos medioambientales globales y para las amenazas de los recursos naturales que afectan a Nueva Zelanda; cuidar la seguridad 
de los neozelandeses y de Nueva Zelanda; crear y aprovechar relaciones internacionales específicas para lograr los objetivos planteados (New Zealand Foreign, 2018a).

Asimismo, el documento establece las prioridades del gobierno neozelandés en materia de política exterior, específicas para 2022. Algunas de éstas son: implementar y capitalizar el Acuerdo (integral y progresivo) de Asociación Transpacífico (TPP-11), especialmente con México, Japón, Canadá y Perú, en 2019; concluir las negociaciones relacionadas con la actualización del Tratado de Libre Comercio con China y la Alianza del Pacífico con la Asociación Económica Integral Regional (RCEP); con la revisión general y la actualización del acuerdo de libre comercio ASEAN-Australia-Nueva Zelanda (AANZFTA), y el Tratado de Libre Comercio con la Unión Europea. Avanzar en las negociaciones bilaterales de los acuerdos comerciales con la India, y en el acuerdo comercial con el Consejo de Cooperación del Golfo hasta su entrada en vigor. Desarrollar y liderar la creación de una agenda progresiva e inclusiva de "Comercio para Todos" durante 2018-2019, e implementar nuevas iniciativas en política comercial para 2020.

\section{ECONOMÍA}

La economía australiana, considerada como de tamaño medio, está intensamente orientada al este asiático. Por casi tres décadas consecutivas Australia ha logrado mantener un crecimiento económico producto, de acuerdo con un informe de la Organización para la Cooperación y Desarrollo Económico (OCDE), de una política macroeconómica fuerte, la implementación de reformas estructurales y el nivel de precios de los productos básicos (cuadro 1). Otros factores como el crecimiento económico mundial, la mejora de los términos de intercambio comercial, el consumo del gobierno y la inversión en infraestructura, también han contribuido a este crecimiento, el cual se espera que se mantenga durante el 2018 a una tasa de 3\%, aproximadamente (OCDE, 2018). Junto con lo anterior, se prevé que tanto las exportaciones como la inversión tengan un impacto positivo en el crecimiento de la economía australiana, mientras que el consumo tendrá uno más moderado. Asimismo, también se espera que la inflación aumente de manera gradual.

Grosso modo, el nivel de vida y de bienestar en Australia son altos. El aumento del empleo ha ayudado a impulsar el nivel de ingresos y el consumo. Asimismo, el nivel salarial está aumentando de manera gradual, por lo que la inflación aún se mantiene por debajo del 
rango objetivo. No obstante, existen cuestionamientos sobre la capacidad de la economía para absorber el nivel de crecimiento del empleo.

En resumen, el país enfrenta varios retos: la incertidumbre propia de los mercados mundiales de productos básicos, de los cuales depende parte de la economía; la desaceleración de la economía china y el objetivo del gobierno chino de reequilibrarla; el alto nivel de endeudamiento de los hogares australianos y la posibilidad de una corrección inesperada en los precios de la vivienda, que podrían reducir el ingreso de los hogares y el nivel de consumo y, simultáneamente, afectar el sector de la construcción (OCDE, 2018). Asimismo, existen otros temas de preocupación para la economía, como la brecha de género existente, la emisión de gases de efecto invernadero y el envejecimiento de la población (Economic Survey of Australia, 2018).

CUADRO 1. Principales indicadores económicos de Australia

\begin{tabular}{|l|r|}
\hline Indicador económico & \multicolumn{1}{|c|}{2017} \\
\hline PIB per cápita (dólares estadounidenses) & 53800 \\
\hline Crecimiento del PIB per cápita (\% anual) & 0.3 \\
\hline PIB (billones) & 1323 \\
\hline Crecimiento del PIB (\% anual) & 21.3 \\
\hline Exportaciones de bienes y servicios (\% del PIB) & 20.6 \\
\hline Importaciones de bienes y servicios (\% del PIB) & 1.3 \\
\hline Inflación, precios al consumidor (\% anual) & 13 \\
\hline $\begin{array}{l}\text { Posición en el ranking mundial de economías } 2017 \\
\text { (por PIB) }\end{array}$ & 554 \\
\hline Comercio mundial 2017 (mil millones de dólares)
\end{tabular}

FUENTE: Elaboración propia con información del Banco Mundial y del Departamento de Asuntos Exteriores y Comercio de Australia.

Por su parte, Nueva Zelanda cuenta con una economía de mercado dependiente en gran medida del comercio internacional y de su cercana relación con Australia, su principal socio comercial. Las industrias más relevantes se centran en el turismo y en las exportaciones de productos agrícolas, sus principales fuentes de crecimiento económico. 
Ahora bien, al igual que la economía australiana, la neozelandesa también tuvo un crecimiento positivo (cuadro 2). De acuerdo con la OCDE, se espera que la tasa de crecimiento de 3\% alcanzada en 2017 se mantenga en 2018 y 2019. Al mismo tiempo, se pronostica una disminución en el crecimiento del consumo interno; las causas son varias: un nivel de inmigración menor ocasionado por los controles de elegibilidad más estrictos para obtener visas de trabajo y el regreso de inmigrantes temporales a sus lugares de origen; la disminución de las exportaciones a finales de 2017 e inicios de 2018, y la debilidad de la inversión privada, la cual se espera aumente en el corto plazo junto con la inversión gubernamental en infraestructura (OCDE, 2018a).

A lo largo de 2018, el nivel de desempleo continuó a la baja; sin embargo, no ha existido un crecimiento salarial considerable, en parte debido a los flujos migratorios de los trabajadores de sectores que enfrentan escasez de mano de obra. Se espera que el aumento de 5\% del salario mínimo por hora establecido en abril de 2018 apoye el crecimiento del ingreso. No obstante, la misma OCDE señala que dicho aumento sólo afectará de manera directa a 3\% de la fuerza laboral (OCDE, 2018a).

Por otra parte, la OCDE considera que mejorará el equilibrio de la política macroeconómica neozelandesa y, en gran medida, que se reducirán las presiones del mercado inmobiliario gracias a los aumentos proyectados de la tasa de interés y del gasto gubernamental. 
CUADRO 2. Principales indicadores económicos

de Nueva Zelanda

\begin{tabular}{|l|r|}
\hline \multicolumn{1}{|c|}{ Indicador económico } & 2017 \\
\hline PIB per cápita (dólares estadounidenses) & 42940 \\
\hline Crecimiento del PIB per cápita (\% anual) & 0.9 \\
\hline PIB (miles de millones) & 205853 \\
\hline Crecimiento del PIB (\% anual) & 3 \\
\hline Exportaciones de bienes y servicios (\% del PIB) & 25.8 \\
\hline Importaciones de bienes y servicios (\% del PIB) & 25.5 \\
\hline Inflación, precios al consumidor (\% anual) & 1.1 \\
\hline Posición en el ranking mundial de economías 2017 (por PIB) & 50 \\
\hline Comercio mundial 2017 (miles de millones de dólares, marzo 2017) & 137.8 \\
\hline
\end{tabular}

FUENTE: Elaboración propia con información del Banco Mundial y del Departamento de Asuntos Exteriores y Comercio de Nueva Zelanda.

\section{COMERCIO EXTERIOR}

En materia de comercio exterior, según informe publicado por el Ministerio de Asuntos Exteriores y Comercio australiano en julio de 2018, el total del comercio de bienes y servicios de Australia alcanzó un total de 554 mil millones de dólares en 2017, ${ }^{7}$ lo cual representó un crecimiento de 11.2 puntos porcentuales respecto a 2016. Mientras que el valor de las exportaciones en bienes y servicios aumentó 14.8\% (280.86 mil millones de dólares) gracias a un incremento en las exportaciones de minerales y combustibles, bienes rurales, servicios y manufacturas, el valor de las importaciones de bienes y servicios aumentó 7.4\% (273.52 mil millones de dólares) en 2017 (Department of Foreign Affairs, 2017, 2018: 5). El aumento ocurrió en bienes de capitales, bienes intermedios y bienes y servicios de consumo.

${ }^{7}$ Los informes del gobierno de Australia utilizan el dólar australiano para fines de este estudio, y para su mejor comprensión realicé la conversión al tipo de cambio del dólar estadounidense (donde 1 dólar australiano equivale a 0.73 de dólar estadounidense). Esto aplica para todos los valores mencionados en el texto. 
Para 2017 China continuó siendo el principal socio comercial de Australia, posición que mantiene desde 2009, con un intercambio bilateral de 133.20 mil millones de dólares en 2017, lo cual representa $24 \%$ del total del comercio australiano. Japón, por su parte, se convirtió en su segundo socio comercial, con una participación de $9.4 \%$, con lo que desplazó a Estados Unidos a la tercera posición, con 9 por ciento.

Por otra parte, cabe destacar que para finales de 2017 Nueva Zelanda registró un superávit comercial de 4.2 mil millones de dólares. Australia sigue siendo su principal socio comercial, aunque para finales de 2017 China se convirtió en el principal destino de las exportaciones de bienes y servicios neozelandeses, siendo las exportaciones de lácteos - en especial la leche en polvo- las más relevantes. Las exportaciones de bienes y servicios a China representaron 15 mil millones de dólares a finales de 2017. Asimismo, la Unión Europea constituyó la principal fuente de importaciones, registrando un valor de $13.4 \mathrm{mil}$ millones de dólares al concluir 2017 (StatsNZ, 2018).

Ambos países cuentan con una economía abierta y multilateral. Durante 2018, Australia mantuvo negociaciones para establecer un acuerdo de libre comercio con la Unión Europea, el Consejo de Cooperación del Golfo, ${ }^{8}$ Hong Kong, la India, la Asociación Económica Integral Regional, la Alianza del Pacífico, el Acuerdo de Comercio de Servicios y el Acuerdo sobre Bienes Ambientales, hasta ahora la agenda comercial australiana más ambiciosa. Asimismo, Australia tiene tratados de libre comercio con Nueva Zelanda, Singapur, Tailandia, Estados Unidos, Chile, la ASEAN, ${ }^{9}$ Malasia, Corea del Sur, Japón y China.

Australia también ha negociado otros acuerdos, los cuales aún no entran en vigor, como el Acuerdo de Asociación Económica entre Indonesia y Australia (IA-CEPA, por sus siglas en inglés), cuyas negociaciones concluyeron el 31 de agosto de 2018; el Acuerdo Integral y Progresivo para la Asociación Transpacífico, firmado el 8 de marzo de 2018 en Santiago de Chile; el Acuerdo de Libre Comercio Perú-Australia, firmado el 12 de febrero de 2018, y el Acuerdo del Pacífico sobre Relaciones Económicas más Estrechas (PACER Plus, por sus siglas en inglés), firmado en junio de 2017. Unidos.

${ }^{8}$ El Consejo es conformado por Bahréin, Kuwait, Omán, Catar, Arabia Saudita y los Emiratos Árabes ${ }^{9}$ Indonesia, Malasia, Filipinas, Singapur, Tailandia, Brunei, Vietnam, Laos, Myanmar y Camboya. 
Por su parte, Nueva Zelanda posee acuerdos comerciales con China, Australia, la ASEAN junto con Australia —el cual pretende actualizar-, Hong Kong, Malasia, Tailandia, Singapur, Corea del Sur, y con Chile, Brunei y Singapur mediante la Asociación Económica Estratégica Transpacífico (2005). También cuenta con otros acuerdos, los cuales, aunque ya se han concluido las negociaciones, aún no entran en vigor, tales como el Acuerdo del Pacífico sobre Relaciones Económicas más Estrechas, el Acuerdo Integral y Progresivo para la Asociación Transpacífico, el Acuerdo Comercial Antifalsificación, y un acuerdo comercial con los países del Consejo de Cooperación del Golfo. Asimismo, mantiene negociaciones comerciales con la India, la Unión Europea, la Alianza del Pacífico, la Asociación Económica Integral Regional y la Unión Aduanera de Rusia, Bielorrusia y Kazajstán.

\section{APÉNDICES}

\section{Apéndice 1. Australia}

Nombre oficial

Capital

Extensión territorial (miles de $\mathrm{km}^{2}$ )

Población (marzo de 2018)

Religión

Idioma

Moneda

Gobierno

Jefe de Estado

Jefe de Gobierno

Principales organizaciones políticas
Mancomunidad de Australia

Canberra

7692

24899100 habitantes

No existe religión de Estado

Inglés

Dólar australiano

Democracia parlamentaria

La reina Isabel II, cuyo representante es el gobernador general Peter Cosgrove desde 2014

El primer ministro Scott Morrison desde agosto de 2018

El Partido Liberal es una de las principales fuerzas políticas, liderado por el primer ministro Morrison. El Partido Laborista Australiano es el principal partido de oposición, liderado por Bill Shorten desde 2013. Asimismo, el Partido Verde, el Partido Nacional de Australia y el Nick Xenophon Team han cobrado importancia en el escenario político 
Ministros clave:

Primer viceministro, ministro de Infraestructura y

Transporte y líder del Partido Nacional

Ministra de Asuntos Internacionales

Ministro de Comercio, Turismo e Inversión

Fiscal general

Ministro de Defensa

Ministro de Finanzas y Servicios Públicos, líder del gobierno en el Senado

Ministra del Medio Ambiente

Tesorero

Ministra de Agricultura y Recursos Hídricos

Ministro de Recursos y Australia del Norte
Michael McCormack

Marise Payne desde agosto de 2018

Simon Birmingham desde agosto de 2018

Christian Porter

Christopher Pyne desde agosto de 2018

Mathias Cornmann

Melissa Price desde agosto de 2018

Josh Frydenberg desde agosto de 2018

David Littleproud

Matthew Cannavan

FUENTE: Elaboración propia con información de la Australian Bureau of Statistics, el Australian Government, y el Department of Foreign Affairs and Trade.

\section{Apéndice 2. Nueva Zelanda}

Nombre oficial

Capital

Extensión territorial $\left(\mathrm{km}^{2}\right)$

Población (junio de 2018)

Religión

Idiomas

Moneda
Nueva Zelanda o Nueva Zelandia

Wellington

267710.0

4885300 habitantes

No existe religión de Estado

Inglés, maorí, y el lenguaje de señas de Nueva Zelanda

Dólar neozelandés 
Gobierno

Jefe de Estado

Jefe de Gobierno

Principales organizaciones políticas

Ministros clave:

Primer viceministro

Finanzas

Asuntos Internacionales

Defensa

Medio Ambiente

Desarrollo Económico

Asuntos Maorís

Justicia

Fiscal general
Democracia parlamentaria

La reina Isabel II, cuyo representante es la gobernadora general Patsy Reddy desde el 28 de septiembre de 2016

La primera ministra Jacinda Ardern desde el 26 de octubre de 2017. Su predecesor, Bill English

Partido Nacional, Partido Laborista, Partido Maorí, ACT Nueva Zelanda, Partido Verde, Nueva Zelanda Primero, Futuro Unido
Grant Robertson

Winston Peters

Ron Mark

David Parker

David Parker

Nanaia Mahuta

Andrew Little

David Parker

FUENTE: Elaboración propia con información del Banco Mundial, New Zealand Foreign Affairs and Trade, y Stats New Zealand Government.

\section{REFERENCIAS BIBLIOGRÁFICAS}

Ainge Roy, E. (2017), "New Zealand Labour Signs Coalition Deal and Makes Winston Peters Deputy PM", The Guardian, 24 de agosto. Recuperado de: https://www.theguardian.com/world/2017/oct/24/new-zealand-kingmaker-winstonpeters-deputy-prime-minister-foreign

Ardern, J. (2017), "Foundation for Strong and Proactive Government", Labor, 24 de octubre. Recuperado de: https://www.labour.org.nz/foundation_for_strong_and_proactive_government 
Australian Council for International Development (2018), Rising Up the Arms Dealers'top 100 is the Wrong Priority. Recuperado de: https://acfid.asn.au/media-releases/risingarms-dealers'-top-100-wrong-priority

Australia Electoral Commission (2018), 2018 By-Elections. Recuperado de: https://www.aec.gov.au/by-elections-2018/

Australian Government (2017), Foreign Policy White Paper. Recuperado de: https://www.fpwhitepaper.gov.au

Australian Government (2018), Report on the Implementation of Sustainable Development Goals. Recuperado de: https://dfat.gov.au/aid/topics/development-issues/2030agenda/Documents/sdg-voluntary-national-review.pdf

Australia's States Offer to Make Treaties with Aboriginals (2018), The Economist, 5 de julio. Recuperado de: https:/www.economist.com/asia/2018/07/05/australias-states-offerto-make-treaties-with-aboriginals

Baidawi, A. (2018), “Australia’s Dual-Citizenship Contagion Claims 5 More Politicians”. The New York Times, 9 de mayo. Recuperado de: https://www.nytimes.com/2018/05/09/world/australia/australia-dual-citizenshippolitician-resign-crisis.html

Banco Mundial (2018), Gross Domestic Product, Recuperado de: http://databank.worldbank.org/data/download/GDP.pdf

Department of Defence. Australian Government (2018), Defense Export Strategy-Fact Sheet. Recuperado de: http://www.defence.gov.au/Export/Strategy/documents/DefenceExportStrategyFactSheet.pdf

Department of Foreign Affairs and Trade, Australian Government (2018), Composition of Trade Australia 2017. Recuperado de: https://dfat.gov.au/aboutus/publications/Documents/cot-cy-2017.pdf

Doran, M. (2018), "Will we Actually Vote on Changing the Constitution after the Dual Citizenship Fiasco", $A B C$ News, 16 de mayo. Recuperado de: 
http://www.abc.net.au/news/2018-05-17/section-44-committee-recommendationsafter-citizenship-fiasco/9770606

Murphy, T. (2017), "Big Vote in Favour of Euthanasia Bill”, Newsroom, 14 de diciembre. Recuperado de: https://www.newsroom.co.nz/2017/12/13/68408/big-vote-in-favourof-euthanasia-bill

New Zealand Foreign Affairs and Trade (2018), Our Relationship with Australia. Recuperado de: https://www.mfat.govt.nz/en/countries-and-regions/australia/\#trade

New Zealand Foreign Affairs and Trade (2018a), Strategic Intentions 2018-2022. Recuperado de: https://www.mfat.govt.nz/assets/MFAT-Corporatepublications/MFAT-Strategic-Intentions-2018-2022.pdf

New Zealand Foreign Affairs and Trade (2017), Strategic Intentions 2018-2021. Recuperado de: https://www.mfat.govt.nz/sistem/files/comounty/publications/Statement/-ofStrategic-Intentions-2017-2021-ruisisty-of-health.pdf

Organización para la Cooperación y el Desarrollo Económicos (2018), Australia-Economic Forecast Summary. Recuperado de: http://www.oecd.org/economy/australiaeconomic-forecast-summary.htm

Organización para la Cooperación y el Desarrollo Económicos (2018a), New ZealandEconomic Forecast Summary. Recuperado de: http://www.oecd.org/newzealand/newzealand-economic-forecast-summary.htm

StatsNZ (2018), Global New Zealand: Year ended December 2017. Recuperado de: https://www.stats.govt.nz/reports/global-new-zealand-year-ended-december-2017 